\title{
Intracranial-to-intracranial bypass for posterior inferior cerebellar artery aneurysms: options, technical challenges, and results in 35 patients
}

\author{
Adib A. Abla, MD, Cameron M. McDougall, MD, Jonathan D. Breshears, MD, and \\ Michael T. Lawton, MD
}

Department of Neurological Surgery, University of California, San Francisco, California

OBJECTIVE Intracranial-to-intracranial (IC-IC) bypasses are alternatives to traditional extracranial-to-intracranial (ECIC) bypasses to reanastomose parent arteries, reimplant efferent branches, revascularize branches with in situ donor arteries, and reconstruct bifurcations with interposition grafts that are entirely intracranial. These bypasses represent an evolution in bypass surgery from using scalp arteries and remote donor sites toward a more local and reconstructive approach. IC-IC bypass can be utilized preferentially when revascularization is needed in the management of complex aneurysms. Experiences using IC-IC bypass, as applied to posterior inferior cerebellar artery (PICA) aneurysms in 35 patients, were reviewed.

METHODS Patients with PICA aneurysms and vertebral artery (VA) aneurysms involving the PICA's origin were identified from a prospectively maintained database of the Vascular Neurosurgery Service, and patients who underwent bypass procedures for PICA revascularization were included.

RESULTS During a 17-year period in which 129 PICA aneurysms in 125 patients were treated microsurgically, 35 ICIC bypasses were performed as part of PICA aneurysm management, including in situ p3-p3 PICA-PICA bypass in 11 patients (31\%), PICA reimplantation in 9 patients $(26 \%)$, reanastomosis in 14 patients $(40 \%)$, and $1 \mathrm{~V}_{3}$ VA-to-PICA bypass with an interposition graft (3\%). All aneurysms were completely or nearly completely obliterated, $94 \%$ of bypasses were patent, $77 \%$ of patients were improved or unchanged after treatment, and good outcomes (modified Rankin Scale $\leq 2$ ) were observed in $76 \%$ of patients. Two patients died expectantly. Ischemic complications were limited to 2 patients in whom the bypasses occluded, and permanent lower cranial nerve morbidity was limited to 3 patients and did not compromise independent function in any of the patients.

CONCLUSIONS PICA aneurysms receive the application of IC-IC bypass better than any other aneurysm, with nearly one-quarter of all PICA aneurysms treated microsurgically at our center requiring bypass without a single EC-IC bypass. The selection of PICA bypass is almost algorithmic: trapped aneurysms at the PICA origin or $p 1$ segment are revascularized with a PICA-PICA bypass, with PICA reimplantation as an alternative; trapped p2 segment aneurysms are reanastomosed, bypassed in situ, or reimplanted; distal p3 segment aneurysms are reanastomosed or revascularized with a PICA-PICA bypass; and aneurysms of the p4 segment that are too distal for PICA-PICA bypass are reanastomosed. Interposition grafts are reserved for when these 3 primary options are unsuitable. A constructive approach that preserves the PICA with direct clipping or replaces flow with a bypass when sacrificed should remain an alternative to deconstructive PICA occlusion and endovascular coiling when complete aneurysm occlusion is unlikely.

http://thejns.org/doi/abs/10.3171/2015.5.JNS15368

KEY WORDS aneurysm; bypass; posterior inferior cerebellar artery; intracranial; vascular disorders

I NTRACRANIAL-TO-INTRACRANIAL (IC-IC) bypasses are alternatives to traditional extracranial-to-intracranial (EC-IC) bypasses to reanastomose parent arteries, reimplant efferent branches, revascularize efferent branches with in situ donor arteries, and reconstruct bifurcated anatomy with interposition grafts that are entirely intracranial. ${ }^{25}$ These bypasses represent an evolution in bypass surgery from using scalp arteries and remote donor sites in the neck toward a more local and reconstructive approach, or, in other words, a more minimally invasive approach to by-

ABBREVIATIONS DSA = digital subtraction angiography; EC-IC = extracranial-to-intracranial; IC-IC = intracranial-to-intracranial; $m$ RS = modified Rankin Scale; PICA = posterior inferior cerebellar artery; RAG = radial artery interposition graft; VA = vertebral artery.

SUBMITTED February 14, 2015. ACCEPTED May 7, 2015.

INCLUDE WHEN CITING Published online November 13, 2015; DOI: 10.3171/2015.5.JNS15368. 
pass. In an analysis of IC-IC bypass as part of the management of complex brain aneurysms conducted 7 years ago, we compared our IC-IC bypass results in 35 patients with those of EC-IC bypass in 47 patients and found comparable bypass patency rates, aneurysm obliteration rates, and patient outcomes. ${ }^{25}$ We have since adopted a practice that utilizes IC-IC bypass preferentially when revascularization is needed in the management of complex aneurysms.

No aneurysm epitomizes this practice more than the posterior inferior cerebellar artery (PICA) aneurysm. PICA aneurysms account for only 3\% to $4 \%$ of brain aneurysms, ${ }^{1,24}$ but they have an unusually high incidence of nonsaccular or fusiform morphology, making them less likely to be clippable. In addition, PICA aneurysms are frequently located distally on the parent artery, rather than arising at the branch sites on the parent artery, which differs significantly from aneurysms at other sites. ${ }^{24}$ In our review of nearly 1700 aneurysms that were treated microsurgically, the incidence of distal aneurysms on the PICA was 5 times that of distal aneurysms on cerebral arteries, accounting for $35 \%$ of all PICA aneurysms as compared with only $6.5 \%$ of all cerebral artery aneurysms. ${ }^{24}$ This same review identified the $\mathrm{p} 2$ and $\mathrm{p} 3$ segments of the PICA as the most common sites of distal PICA aneurysms, which are segments notable for their curvature in the caudal or infratonsillar loop. ${ }^{24}$ Furthermore, PICA aneurysms have an increased frequency of complicating features, like intraluminal thrombosis, giant size, and coil recurrence. In the senior author's previously published experiences, PICA/vertebral artery (VA) aneurysms classified as thrombotic, giant, or coiled were observed in 7\%,11 $6.3 \%,{ }^{27}$ and $7 \%{ }^{28}$ of patients, respectively, which exceeded the average incidence of PICA aneurysms.

This report describes our experience with IC-IC bypass as applied to PICA aneurysms in 35 patients and exemplifies this evolution in bypass surgery. In addition, this experience also demonstrates that the combination of unclippable pathology associated with PICA aneurysms and the frequent application of revascularization techniques make open surgery an important treatment option for these lesions. Various bypass techniques are analyzed in detail in order to develop an algorithm for managing PICA aneurysms and selecting the best bypass option.

\section{Methods \\ Patients}

This study was approved by the Institutional Review Board and conducted in compliance with Health Insurance Portability and Accountability Act regulations. Patients with PICA and VA aneurysms were identified from a prospectively maintained database of the Vascular Neurosurgery Service, and those patients who underwent bypass procedures for PICA revascularization were included. Medical records, including preoperative and postoperative radiographic imaging, operative reports, intraoperative photographs, hospital course, and outpatient visits, were reviewed retrospectively. Clinicians who were not directly involved in the patients' care (J.D.B. and C.M.M.) performed all outcome assessments using the modified Rankin Scale (mRS).

A numerical nomenclature was used to describe the
PICA segments: p1 denoting the anterior medullary segment; p2, lateral medullary segment; p3, tonsillomedullary segment, including the caudal loop; p4, telovelotonsillar segment, including the cranial loop; and p5, cortical segment. The numerical nomenclature for the VA segments included: $\mathrm{V}_{3}$, the extradural segment from the $\mathrm{C}-2$ transverse foramen to the foramen magnum dura; and $\mathrm{V}_{4}$, intradural segment from the dura to the vertebrobasilar junction.

\section{Bypass Techniques}

Bypasses were performed only when conventional clipping failed, as due to complex branch anatomy, giant size, dolichoectatic morphology, intraluminal thrombus, or atherosclerotic neck tissue. Bypass was indicated for aneurysm trapping that occluded the PICA or a dominant VA. Balloon test occlusion was not useful for selecting patients for bypass because of difficulties cannulating the PICA with the balloon-tipped catheter and the high false-positive rate associated with test occlusion of the parent VA due to balloon occlusion of the perforators originating from the $\mathrm{V}_{4}$ segment. Intraoperative test occlusion using neurophysiological monitoring was not useful either because of the insensitivities of routine motor and somatosensory evoked potentials and the resulting high false-negative rate. Therefore, a "universal" rather than "selective" approach to revascularization was employed.

Bypass options included: 1) in situ bypass with p3-p3 PICA-PICA bypass; 2) reimplantation of the PICA to the proximal $\mathrm{V}_{4}$ segment of the VA; 3) PICA reanastomosis after aneurysm excision; and (4) high-flow bypass from the $\mathrm{V}_{3}$ segment of the VA to PICA with radial artery interposition graft (RAG) (Fig. 1). In situ bypass required donor and recipient arteries that lie in parallel and close to one another, and the caudal loops of the p3 segments bilaterally met this requirement in the midline just below the obex of the fourth ventricle. These loops required detachment from their arachnoidal adhesions in order to bring them together freely. The anastomosis was side-to-side, with the first suture line sewn intraluminally.

Reimplantation was needed when clipping closed the neck and excluded the aneurysm, preserving the VA but sacrificing the PICA. Reimplantation was also needed for trapped dolichoectatic VA aneurysms involving the PICA. The occluded PICA was reconstituted by transecting it at its origin, transposing it within the vagoaccessory triangle from the suprahypoglossal triangle to the infrahypoglossal triangle, and reimplanting it proximally to the $\mathrm{V}_{4}$ segment. The anastomosis was end-to-side, analogous to a temporal artery-to-middle cerebral artery bypass.

Reanastomosis reconnected the transected ends of the afferent and efferent parent arteries after trapping or excising the aneurysm and completely detaching the afferent and efferent arteries. The reconstruction used an end-toend anastomosis, which worked well with PICA aneurysms that are frequently fusiform, small or medium in size, and do not branch in the way that many cerebral arteries do as they course distally. The cut ends of the parent artery were approximated without tension in order to prevent the sutures from tearing through the tissues and ruining the repair. The PICA's tortuosity provided sufficient redundancy 

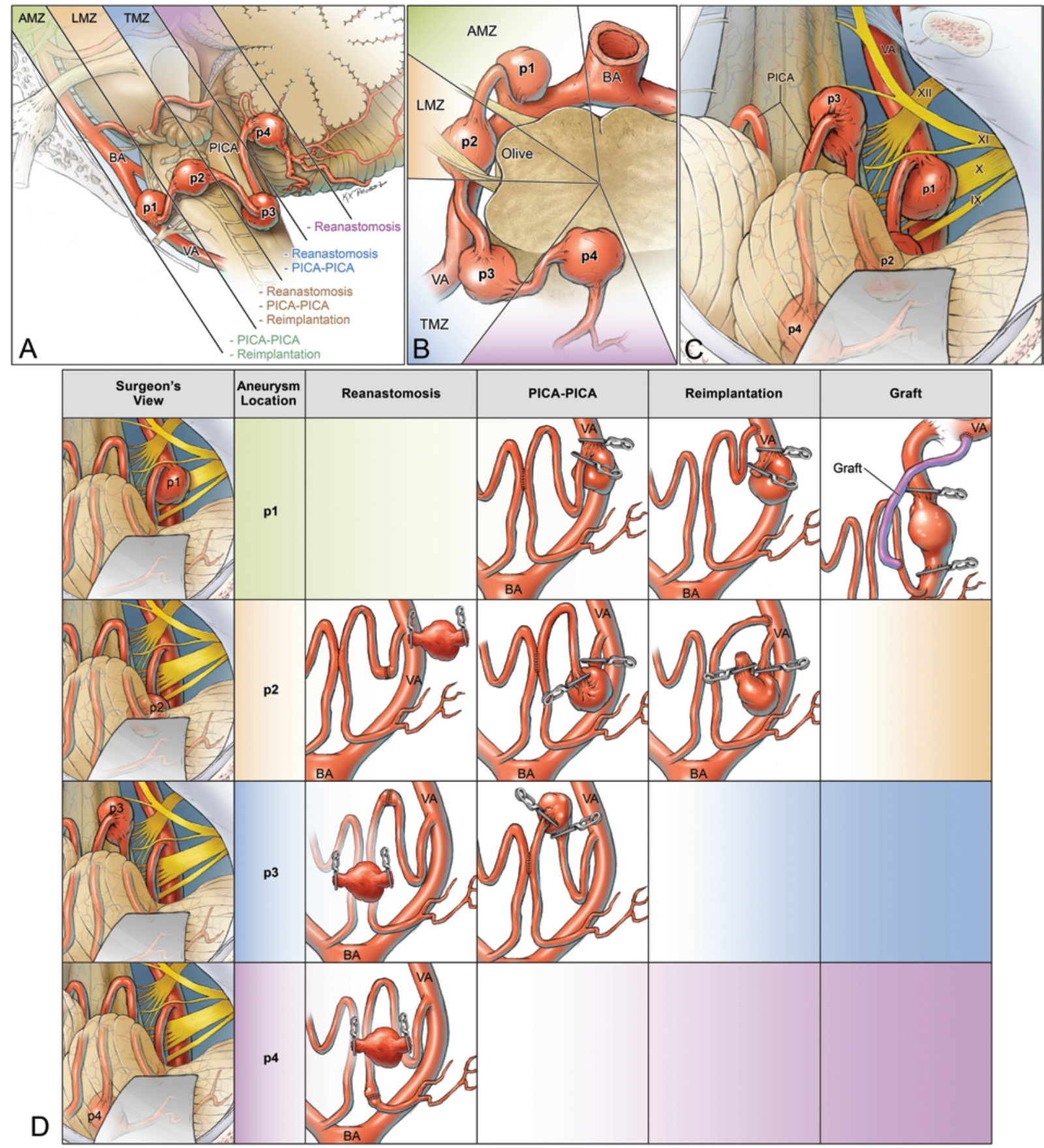

FIG. 1. Algorithm for selecting PICA bypasses. Selection of the bypass technique was influenced by the aneurysm's location according to PICA segments. A: Lateral view. B: Axial cross-sectional view. C: Surgeon's view through a left far lateral craniotomy. D: Table summarizing the bypass options according to segmental anatomy, with $p 1$ and $p 2$ aneurysms having the most options. $\mathrm{AMZ}$ = anterior medullary zone; $\mathrm{BA}=$ basilar artery; $\mathrm{LMZ}=$ lateral medullary zone; $\mathrm{TMZ}=$ tonsillomedullary zone. IX, $\mathrm{X}$, $\mathrm{XI}, \mathrm{XII}=$ cranial nerves. Copyright Michael Lawton. Used with permission.

to approximate the arteries. PICA aneurysms treated with reanastomosis were distally located, which increased the working room for the anastomosis.

Interposition grafts were rarely needed for PICA aneurysms involving a dominant VA that was trapped in the context of a hypoplastic contralateral VA or a contralateral VA that terminated in the PICA without contributing to basilar circulation. The $\mathrm{V}_{3}$ segment provided an excel- lent donor site, and the PICA served as the recipient site. This bypass graft was short and the RAG was matched in caliber, making it preferable over saphenous vein grafts. Preoperative Allen test with Doppler ultrasound ensured adequate collateral circulation to the hand through the ulnar artery and palmar arch. The ipsilateral forearm was accessed for harvesting in the far-lateral position. The RAG was dilated with pressure distension, perfused with 
heparin, and immersed in nitroprusside solution. Unlike other IC-IC bypasses, interposition grafts required 2 anastomoses, which can be end-to-side or end-to-end, and were planned to minimize ischemia time in the posterior circulation.

During the anastomosis, a heparin solution was irrigated locally and evacuated with microvac suction (PMT Corporation). Systemic heparin was not used. The continuous suturing technique was preferred over the interrupted technique in order to reduce the number and amount of time spent tying knots. Bypasses were checked for patency using indocyanine green videoangiography and Doppler ultrasound. During clamping, patients were maintained with barbiturate- or propofol-induced electroencephalography burst suppression with a mildly elevated systolic arterial blood pressure. Postoperative angiography evaluated the patency of the bypass and obliteration of the aneurysm, and patients were maintained on $325 \mathrm{mg}$ acetylsalicylic acid indefinitely.

\section{Results}

\section{Patient and Aneurysm Characteristics}

During the 17-year period from September 1997 to January 2014, 3147 aneurysms were treated microsurgically in 2414 patients as part of a consecutive, single-surgeon series. Among these were 129 PICA aneurysms in 125 patients. Included in this cohort were patients with VA aneurysms that involved the PICA origin, but not VA aneurysms that were proximal or distal to PICA without PICA involvement. In the same time period, 318 bypasses were performed, including 155 bypasses that were performed as part of aneurysm management and 35 IC-IC bypasses performed as part of PICA aneurysm management. Therefore, PICA aneurysms accounted for $4.1 \%$ of all aneurysms and were treated in $5.2 \%$ of all aneurysm patients; $22.6 \%$ of all bypasses for aneurysms involved a PICA aneurysm, and $27.1 \%$ of all treated PICA aneurysms required bypass.

The average patient age was 54.8 years, and there was a male predominance (Table 1). Most patients (69\%) presented with subarachnoid hemorrhage with an average Hunt and Hess Grade of 2.5. The mean mRS at presentation of all patients was 2.3 (range 1-5). Eighteen aneurysms (52\%) were located at or adjacent to the PICA origin ( $\mathrm{p} 1$ and $\mathrm{p} 2$ segments), 7 (20\%) were distal PICA aneurysms (p3 and p4 segments), and 10 (29\%) involved the $\mathrm{V}_{4}$ VA segment and incorporated the PICA origin. There was 1 patient with a mirror PICA aneurysm that was treated simultaneously with contralateral aneurysm clipping, and another patient with 2 distal PICA aneurysms, 1 of which was treated with clipping and the other with reanastomosis. Only 5 PICA aneurysms (14\%) had saccular morphology; instead, most had fusiform morphology (29 aneurysms; 83\%), intraluminal thrombus (15 aneurysms; $43 \%$ ), and large size (mean aneurysm diameter $12.7 \mathrm{~mm}$ ).

\section{Surgical Treatment}

In situ p3-p3 PICA-PICA bypass (Fig. 2) was used in 11 patients (31\%), and PICA reimplantation (Fig. 3) was used in 9 patients (26\%) (Table 2). Reanastomosis after aneurysm excision (Fig. 4) was the most common IC-IC bypass with PICA aneurysms and was used in 14 patients (40\%),
TABLE 1. Summary of patients, PICA aneurysm characteristics, and outcomes

\begin{tabular}{|c|c|}
\hline Characteristic & Value* $^{*}$ \\
\hline No. of patients & 35 \\
\hline \multicolumn{2}{|l|}{ Age in yrs } \\
\hline Mean \pm SD & $54.8 \pm 13.5$ \\
\hline Range & $30-86$ \\
\hline No. of female patients & $16(45.7)$ \\
\hline Subarachnoid hemorrhage & $24(68.6)$ \\
\hline \multicolumn{2}{|l|}{ PICA aneurysm location } \\
\hline $\mathrm{p} 1$ & $10(28.6)$ \\
\hline p2 & $8(22.9)$ \\
\hline p3 & $5(14.3)$ \\
\hline $\mathrm{p} 4$ & $2(5.7)$ \\
\hline VA aneurysm & $10(28.6)$ \\
\hline \multicolumn{2}{|l|}{ PICA aneurysm size in $\mathrm{mm}$} \\
\hline Mean \pm SD & $12.7 \pm 13.4$ \\
\hline Range & $2-50$ \\
\hline Giant aneurysm & $4(11.4)$ \\
\hline \multicolumn{2}{|l|}{ PICA aneurysm morphology } \\
\hline Fusiform & $29(82.9)$ \\
\hline Thrombotic & $15(42.9)$ \\
\hline \multicolumn{2}{|l|}{ Preoperative mRS score } \\
\hline 1 & $16(45.7)$ \\
\hline 2 & $5(14.3)$ \\
\hline 3 & $3(8.6)$ \\
\hline 4 & $8(22.9)$ \\
\hline 5 & $3(8.6)$ \\
\hline Mean \pm SD & $2.3 \pm 1.5$ \\
\hline Range & $1-5$ \\
\hline
\end{tabular}

* Values are shown as number (\%) unless otherwise indicated.

of whom 13 underwent end-to-end PICA reanastomosis and 1 underwent end-to-end $\mathrm{V}_{4}$-PICA reanastomosis. One patient (3\%) underwent $\mathrm{V}_{3}$-to-PICA bypass with an interposition RAG for a dissecting aneurysm that extended up to the PICA in the dominant VA with an atretic contralateral VA. After proximal clip occlusion of the dissecting aneurysm, the bypass supplied the PICA with anterograde flow to the PICA territory distally and retrograde flow to the distal $\mathrm{V}_{4}$ segment (distal to the aneurysm) to restore flow to the basilar circulation.

Twenty-six (74\%) aneurysms were trapped or excised, $4(11 \%)$ aneurysms were occluded proximally, 3 (9\%) aneurysms were occluded endovascularly with either VA sacrifice or aneurysm coiling, and $2(6 \%)$ aneurysms were surgically clipped (Table 3). Bypasses were performed through a far-lateral approach in $31(89 \%)$ patients and a midline suboccipital craniotomy in 4 (11\%) patients.

\section{Outcomes}

Angiographic bypass patency was shown in 32 of 34 patients with available imaging (94\%), and complete aneurysm obliteration was seen in 31 patients (89\%) (Table $3)$. Three additional aneurysms (9\%) had near-complete 

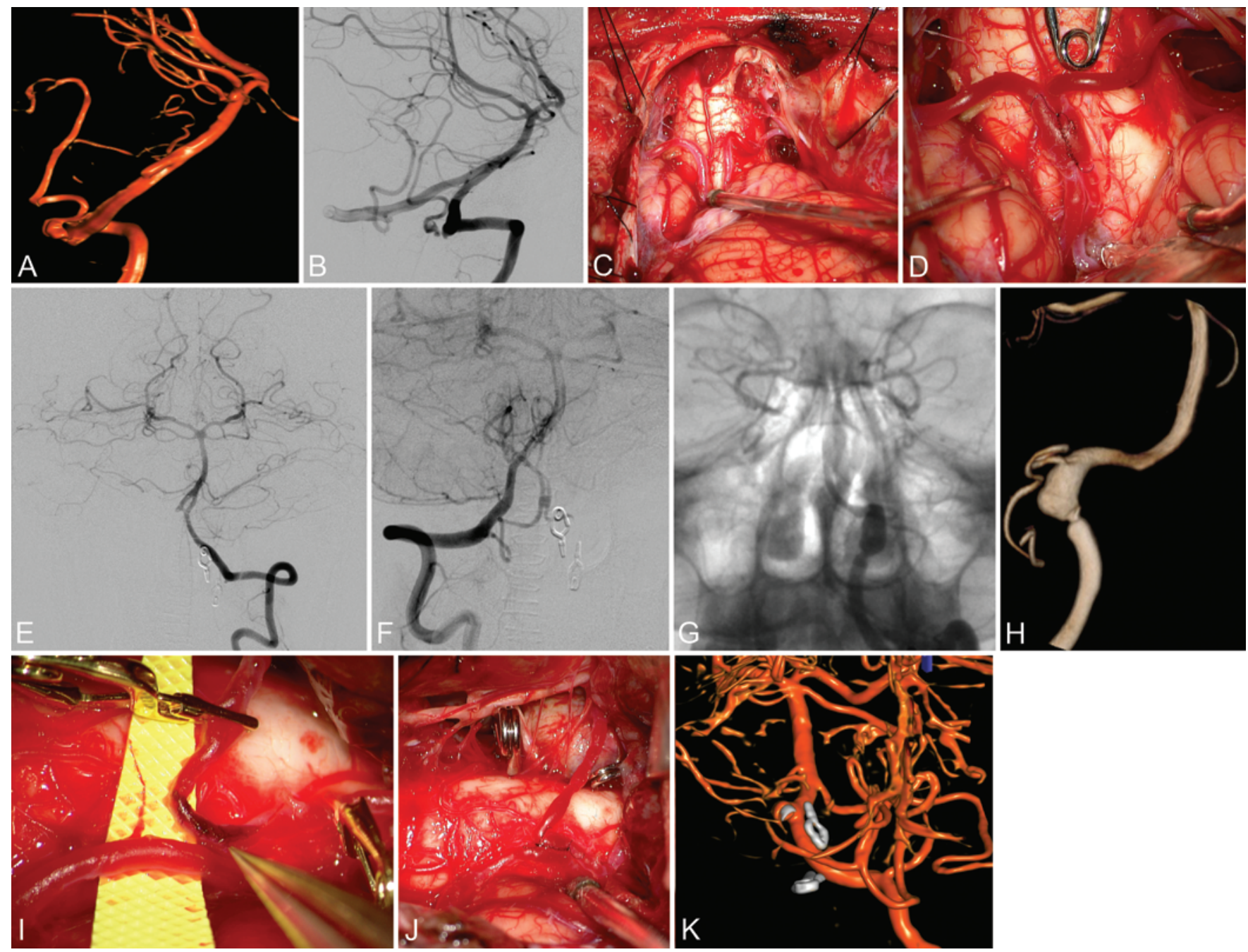

FIG. 2. In situ PICA-PICA bypass. A: A 28-year-old woman presented with subarachnoid hemorrhage from a fusiform aneurysm arising from the left PICA origin and p1 segment, as seen on 3D rotational angiography (left VA injection; lateral view). B: Parallel alignment of the $\mathrm{p3}$ segments, as seen on digital subtraction angiography (DSA) (left VA injection; right anterior oblique view), was ideal for in situ bypass. C: Left far-lateral craniotomy exposed both caudal loops of PICA and their ascending p3 segments, which joined naturally with a side-to-side anastomosis (D). E and F: The aneurysm was trapped and excised (DSA; left VA injection; anteroposterior view), and the contralateral PICA filled the left PICA territory through the anastomosis both antero- and retrograde (DSA; right VA injection; anteroposterior view). G and H: A 50-year-old man presented with headaches without subarachnoid hemorrhage and a fusiform aneurysm involving the left VA (DSA; left VA injection; anteroposterior view) and PICA origin (3D rotational angiogram, posterior view). I: Intraoperatively, the PICA was acutely occluded at the PICA origin. In contrast to the first case, the caudal loops and ascending p3 segments were widely separated and, even after extensive dissection, were in parallel alignment over a short segment. J: This anatomy resulted in $90^{\circ}$ angulation of the left PICA, which can create tension on the arteries, kinking of the afferent limb of the anastomosis, and/or occlusion of the bypass. K: The aneurysm was trapped with clips on the proximal and distal VA and PICA origin, and postoperative 3D rotational angiography demonstrated a patent anastomosis and complete aneurysm exclusion. This case demonstrates the difficulties associated with in situ bypass with PICAs that do not normally lie in parallel.

obliteration, including 2 PICA aneurysms that were coiled in the second stage after PICA-PICA bypass and 1 giant, recurrent, coiled PICA aneurysm that was proximally clip occluded after PICA reimplantation and had not yet thrombosed completely on postoperative angiography.

Ischemic complications were observed in 2 patients (7\%). The donor VA thrombosed in 1 patient after PICA reimplantation and trapping of a ruptured, fusiform PICAVA aneurysm. In another patient with a similar aneurysm, an end-to-end VA-PICA reanastomosis was performed because there was insufficient donor VA proximally for a longitudinal arteriotomy and end-to-side anastomosis. The recipient PICA was fish-mouthed, but the mismatch in caliber between the transected VA and PICA, plus inherent atherosclerotic disease in the VA wall, contributed to a postoperative bypass occlusion (Fig. 3). Both patients developed new inferior cerebellar infarcts.

New lower cranial nerve neuropathies were observed in 5 patients, but they resolved completely in 2 patients and resulted in permanent morbidity in 3 patients $(9 \%)$. Three 

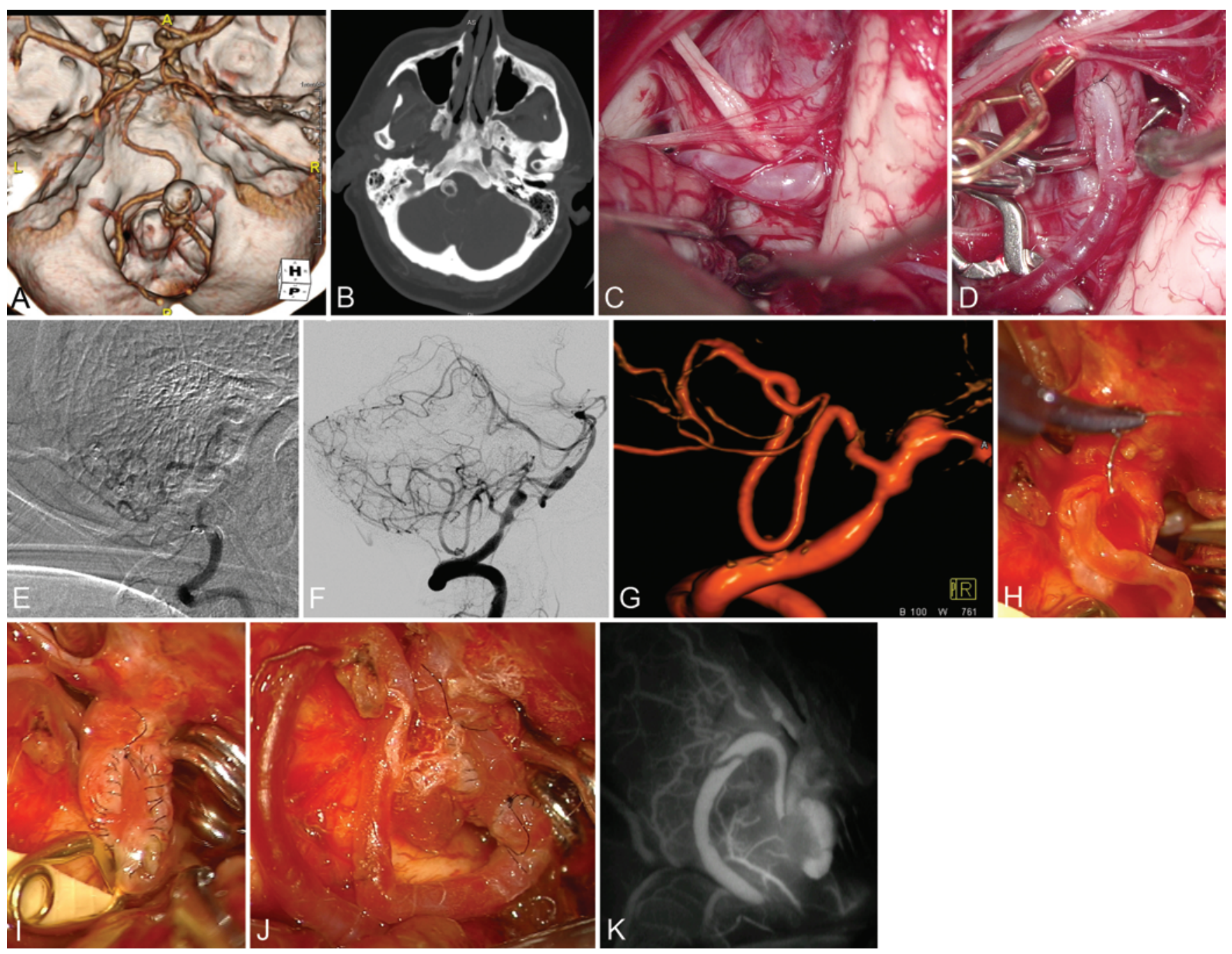

FIG. 3. PICA reimplantation. A: A 58-year-old woman presented with a large, calcified, and thrombotic right PICA aneurysm that failed an attempt at coiling at an outside hospital (CT angiogram with 3D reconstruction, posterior view). B: The PICA originated from the calcified posterior wall of the aneurysm (axial CT angiogram). C: Intraoperatively, the aneurysm could not be clip-occluded proximally without compromising the PICA. D: Therefore, the PICA was transected, mobilized from the aneurysm, and reimplanted on the proximal VA with an end-to-side anastomosis performed in the infrahypoglossal portion of the vagoaccessory triangle. E: This bypass enabled clip occlusion of the aneurysm's inflow, and postoperative angiography demonstrated a reimplanted PICA with complete aneurysm exclusion (DSA; right VA injection; lateral view). F: This 59-year-old woman presented with a subarachnoid hemorrhage from an aneurysm involving the left VA and PICA origin (DSA; left VA injection; lateral view). G: Three-dimensional rotational angiogram (lateral view). H: In contrast to the first reimplantation case, the length of a normal, intradural VA was short and did not accommodate end-to-side reimplantation, even with the proximal temporary clip placed on the extradural VA. Therefore, the VA was transected and the PICA was reimplanted end-to-end. I: Caliber mismatch between the PICA and VA was managed by fish-mouthing the PICA, and, although the anastomosis was initially patent, a kink in the PICA caused it to occlude after 20 minutes. J: The kinked segment was excised, and the PICA was reanastomosed end-to-end just distal to the VA-PICA reimplantation. K: Indocyanine green videoangiography demonstrated PICA patency intraoperatively, but the bypass was occluded on postoperative angiography. This case demonstrates the difficulties associated with PICA reimplantation on a short VA segment.

of these patients received PICA reimplantations, and the others underwent PICA-PICA in situ bypass and reanastomosis. Permanent lower cranial neuropathies resulted in dysphagia and hoarseness and increased the mRS Scores from 1 to 2 in all 3 patients.

Two patients with poor-grade subarachnoid hemorrhage at presentation died expectantly in the perioperative period, 1 after complications from aspiration pneumonia and 1 after presenting comatose with a large posterior fossa subdural hematoma, who never improved postoperatively (surgical mortality 6\%). Two additional patients were lost on late follow-up. Of the remaining patients, $14(40 \%)$ patients improved according to their mRS scores at the last follow-up, 13 (37\%) patients remained unchanged, and 4 (11\%) patients were worse (mean follow-up duration 16 months). The mean mRS score was 1.9 (range 1-6) at the last follow-up. Overall, 25 patients $(76 \%)$ had good outcomes with final $\mathrm{mRS}$ scores $\leq 2$. 
TABLE 2. Summary of IC-IC bypasses for PICA aneurysms

\begin{tabular}{|c|c|c|c|c|c|c|}
\hline Bypass & $\begin{array}{c}\text { No. of } \\
\text { Patients (\%) }\end{array}$ & Donor-Recipient & Graft & Approach & Anastomosis Technique & No. of Anastomoses \\
\hline In situ bypass & $11(31)$ & $\begin{array}{l}\text { PICA-PICA (contralateral } \\
\text { p3-ipsilateral p3) }\end{array}$ & None & Far lateral or suboccipital & Side-to-side & 1 \\
\hline \multirow[t]{2}{*}{ Reanastomosis } & $13(37)$ & PICA-PICA (ipsilateral p2-p4) & None & Far lateral or suboccipital & End-to-end & 1 \\
\hline & $1(3)$ & VA-PICA (ipsilateral V4-p1) & None & Far lateral & End-to-end & 1 \\
\hline Reimplantation & $9(26)$ & VA-PICA (ipsilateral V4-p1) & None & Far lateral & End-to-side & 1 \\
\hline Interpositional & $1(3)$ & VA-PICA (extradural V3-p3) & RAG & Far lateral & End-to-side & 2 \\
\hline
\end{tabular}
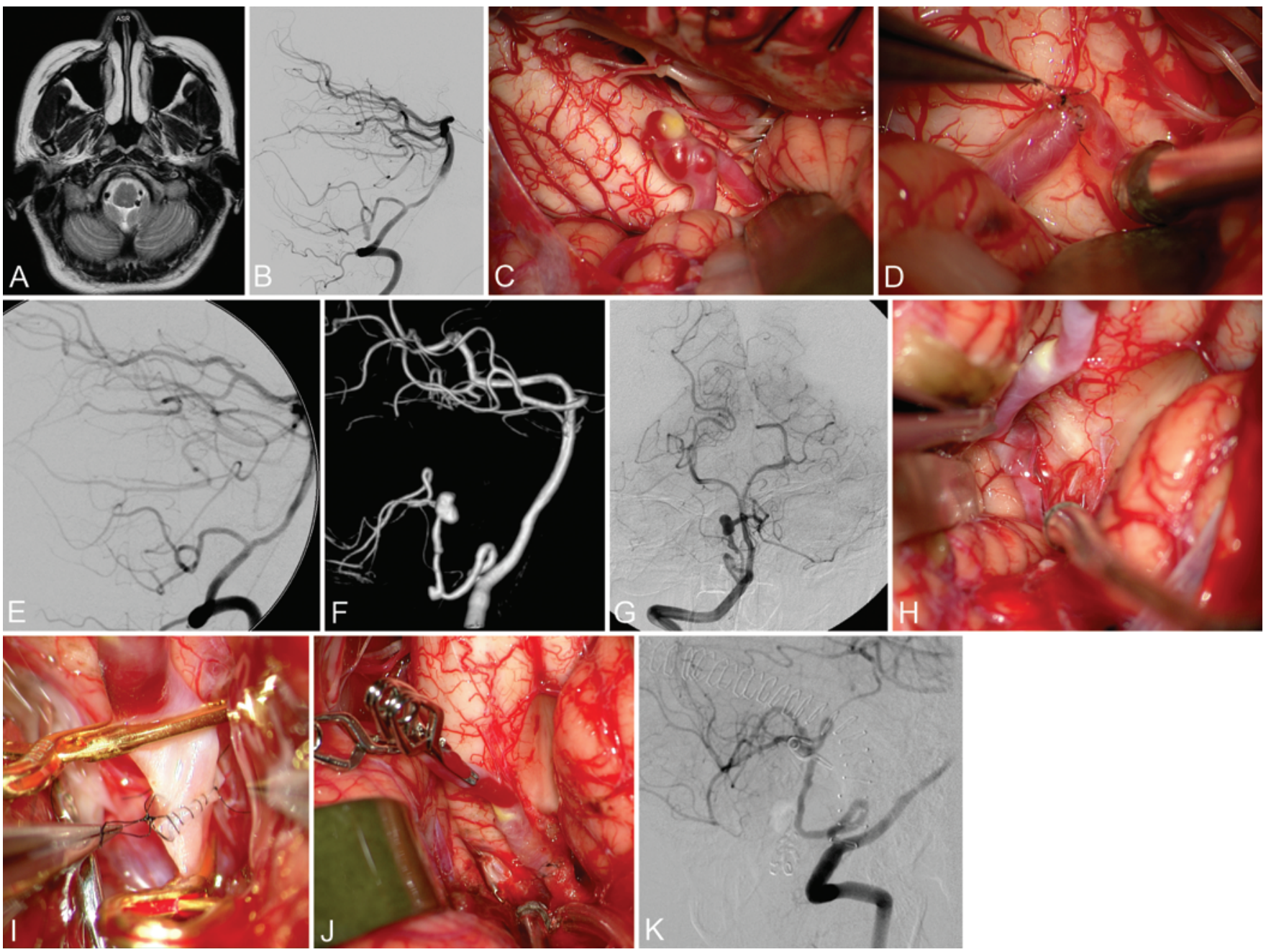

FIG. 4. PICA reanastomosis. A: A 45-year-old woman presented with a distal, fusiform, left PICA aneurysm seen in the tonsillomedullary fissure (axial T2-weighted MR image). B: The aneurysm was located at the caudal PICA loop (p3 segment) with separate inflow and outflow arteries (DSA; left VA injection; lateral view). C and D: The aneurysm was identified beneath the tonsil and excised, after which an end-to-end reanastomosis was completed. E: Postoperative angiography confirmed bypass patency (DSA; left VA injection; lateral view). F: A 39-year-old man presented with 2 distal, fusiform, right PICA aneurysms, 1 at the caudal loop and the larger one at the cranial loop of PICA (3D rotational angiogram; right VA injection; lateral view). G: The left PICA was absent and the distal-most efferent aneurysm supplied both hemispheres (DSA; right VA injection; anteroposterior view). H: In contrast to the first reanastomosis case, this distal PICA aneurysm at the cranial loop (p4 segment) was deeply located near the lateral recess of the fourth ventricle and was exposed through a telovelar approach. I and J: The distal aneurysm was reconstructed with an end-to-end reanastomosis, and the proximal aneurysm was clip reconstructed. K: Postoperative angiography confirmed patency to PICA and the exclusion of both aneurysms (DSA; right VA injection; lateral view). 
TABLE 3. Summary of surgical results and patient outcomes

\begin{tabular}{|c|c|}
\hline Characteristic & Value* $^{*}$ \\
\hline \multicolumn{2}{|l|}{ Craniotomy } \\
\hline Far lateral & $31(88.6)$ \\
\hline Suboccipital & $4(11.4)$ \\
\hline \multicolumn{2}{|l|}{ Aneurysm occlusion } \\
\hline Clipping & $2(5.7)$ \\
\hline Trapping & $26(74.3)$ \\
\hline Proximal clip occlusion & $4(11.4)$ \\
\hline Endovascular occlusion & $3(8.6)$ \\
\hline \multicolumn{2}{|l|}{ Angiographic aneurysm obliteration } \\
\hline Complete & $31(88.6)$ \\
\hline Near complete & $3(8.6)$ \\
\hline No angiographic examination & $1(2.9)$ \\
\hline Bypass patency & $32(94.1)$ \\
\hline Bypass occlusion & $2(5.9)$ \\
\hline \multicolumn{2}{|l|}{ Patient outcomes } \\
\hline Mean follow-up duration (mos) & 16.3 \\
\hline \multicolumn{2}{|l|}{ Final postoperative mRS score } \\
\hline 1 & $21(63.6)$ \\
\hline 2 & $4(12.1)$ \\
\hline 3 & $3(9.1)$ \\
\hline 4 & $3(9.1)$ \\
\hline 5 & $0(0)$ \\
\hline 6 & $2(6.1)$ \\
\hline Lost to late follow-up & 2 \\
\hline Final postoperative mRS, mean \pm SD (range) & $1.9+1.5(1-6)$ \\
\hline \multicolumn{2}{|l|}{ Change in $\mathrm{mRS}$ score } \\
\hline Improved & $14(40.0)$ \\
\hline Same & 13 (37.1) \\
\hline Worse & $4(11.4)$ \\
\hline Dead & $2(5.7)$ \\
\hline
\end{tabular}

* Values are shown as number (\%) unless otherwise indicated.

\section{Discussion}

\section{Bypass Options for PICA Aneurysms}

PICA aneurysms can be used to demonstrate the application of IC-IC bypass techniques better than any other aneurysm. Our experience in 35 patients represents nearly one-quarter of all PICA aneurysms treated microsurgically at our center, and without a single EC-IC bypass. All aneurysms were completely or nearly completely obliterated, $94 \%$ of bypasses were patent, $77 \%$ of patients were improved or unchanged after treatment, and good outcomes $(\mathrm{mRS} \leq 2)$ were observed in $76 \%$ of patients. Ischemic complications were limited to 2 patients in whom bypasses were occluded, and permanent lower cranial nerve morbidity was limited to 3 patients, none of which compromised independent function. We found that IC-IC bypasses match the spectrum of aneurysm pathology in this region, and intracranial donor arteries spare the neurosurgeon the tedious dissection required for occipital artery harvest.

PICA-PICA bypass is the easiest of the IC-IC bypasses.
It is performed in the midline through a far lateral craniotomy and can be done through a standard suboccipital craniotomy when aneurysm access is not needed. Visibility and maneuverability are excellent, and the lower cranial nerves are not involved. The caudal loops of the PICA are generously mobilized to bring the ascending segments together naturally; if they are brought together with sutures under tension, the sutures can tear through the arterial wall or the artery can kink and occlude. A long arteriotomy (2-3 times the diameter of the PICA) ensures flow across the anastomosis. The caliber of the contralateral donor PICA should be equal to or larger than the ipsilateral recipient.

Reanastomosis after aneurysm excision is an elegant technique for the right aneurysm, such as those that are distally located with fusiform morphology, small to medium size, and single afferent and efferent arteries. The endto-end suture line is shorter than the other techniques and requires fewer bites, making it quicker. The artery must be rotated to visualize the suture lines, either by moving the shanks of the temporary clips or providing some tension on the deep suture. Alternatively, the deep suture line can be sewn intraluminally. Three cases with reanastomosis proved difficult: 1 between the VA and PICA due to severe caliber mismatch, and 2 others on distal p4 PICA aneurysms located at the apex of the cranial loop of the PICA where exposure was restricted.

Reimplantation may be the hardest of the IC-IC bypasses. Although aggressive condylectomy opens the surgical corridor, it is deep and surrounded by the lower cranial nerves that form the infrahypoglossal portion of the vagoaccessory triangle. Consequently, the risk of postoperative cranial neuropathy is greatest with this technique. In addition, the risk of postoperative bypass occlusion is also greatest with this technique, occurring in the only 2 patients without a patent graft. The VA wall is more layered than that of the PICA and may be atherosclerotic, creating a mismatch in wall thickness as well as in caliber. The thickness of the VA wall and the irregularities in these layers might increase its thrombogenicity and diminish the patency rate of the anastomosis. Reimplantations at other sites, like with middle cerebral artery aneurysms, with unequal wall thickness between the donor and recipient arteries do not have this problem, and the patency rates there have been much better. The bites of the suture must be full thickness, and inaccuracies in the suturing technique can also threaten the bypass. The end-to-side anastomosis again requires a long arteriotomy in VA, and the corridor is too narrow for an arterial punch. The difficulties with a short VA donor site can be managed by placing a temporary clip on the proximal VA extradurally and lifting the accessory nerve. Temporary occlusion time is well tolerated when the contralateral VA supplies the basilar circulation. The outcomes with PICA reimplantation in this experience have led to the conclusion that this technique should be reserved as an alternative when other IC-IC options, namely in situ PICA-PICA bypass or PICA reanastomosis, are not possible or favorable.

The use of interposition grafts was limited to 1 case involving an aneurysm on the lone VA that contributed to basilar circulation. The extradural $\mathrm{V}_{3}$ VA segment is a robust donor that is exposed with resection of the pos- 
terolateral $\mathrm{C} 1$ vertebral arch and control of the overlying perivertebral venous plexus. Large arteriotomy with an aortic punch opens an excellent donor site, and radial artery grafts are ideally sized. Distal anastomosis to the PICA can supply the PICA alone or the basilar circulation, depending on the aneurysm's anatomy, revascularization requirements, and the location of the distal clips.

The selection of PICA bypass is almost algorithmic (Fig. 1). Trapped aneurysms at the PICA origin or $\mathrm{p} 1 \mathrm{seg}-$ ment are revascularized with PICA-PICA bypass, with PICA reimplantation as an alternative. Trapped aneurysms at the $\mathrm{p} 2$ segment have the most options: reanastomosis, PICA-PICA bypass, or reimplantation. Distal aneurysms at the $\mathrm{p} 3$ segment are excised and reanastomosed, or revascularized with PICA-PICA bypass. Therefore, PICA-PICA bypass is almost always an option, except for aneurysms beyond the $\mathrm{p} 3$ segment. Our findings that bypass patency is lower and cranial nerve morbidity is higher with PICA reimplantation than with other techniques makes this technique a secondary alternative. Aneurysms of the p4 segment that are near to or involve the cranial loop and that are too distal for PICA-PICA bypass are excised/reanastomosed. Interposition grafts are reserved for when these 3 primary options are unsuitable or deliberate occlusion of the dominant or only VA requires the replacement of VA flow.

\section{Bypass Indications}

The PICA territory has collateral connections with the superior cerebellar artery superiorly and the anterior inferior cerebellar artery laterally, calling into question the necessity of PICA revascularization and encouraging deconstructive approaches to PICA aneurysms. While our results do not specifically address these issues, the endovascular literature provides some insights (Table 4). $3,10,16-18,21,23$ The use of stents, stent-assisted coiling, or balloon-assisted coiling improves the rates of PICA preservation, but even in the largest and most recent experience selective aneurysm coiling was possible in 60 of $76(79 \%)$ patients and the PICA was sacrificed deliberately in $11(14 \%)$ patients. PICA occlusion was accompanied by infarcts in $6(8 \%)$ patients, and $4(5 \%)$ patients in the series died in hospital. ${ }^{2}$ These results counter the prevailing opinion that PICA is expendable with minimal morbidity. ${ }^{2,15}$ Deconstructive approaches that sacrifice the PICA can cause medullary and cerebellar infarcts, cerebellar edema, acute hydrocephalus, and brainstem compression. While some of these complications may be tolerable in some cases, they frequently cause deterioration and can require urgent decompressive craniectomy, as in our 2 cases of bypass occlusion. In the absence of hard evidence establishing clinical tolerance to PICA sacrifice, a constructive approach that preserves the PICA with direct clipping or replaces flow with bypass when sacrificed should remain a viable alternative to deconstructive, endovascular PICA occlusion and direct coiling when complete aneurysm occlusion is unlikely to be achieved and the risks of recurrence and retreatment might be significant. ${ }^{2}$ This thinking forms the basis for our "universal" approach to bypass with PICA aneurysms.

In addition to its collateral connections, the PICA territory has considerable anatomical variation that requires careful evaluation when planning IC-IC bypasses. For ex-

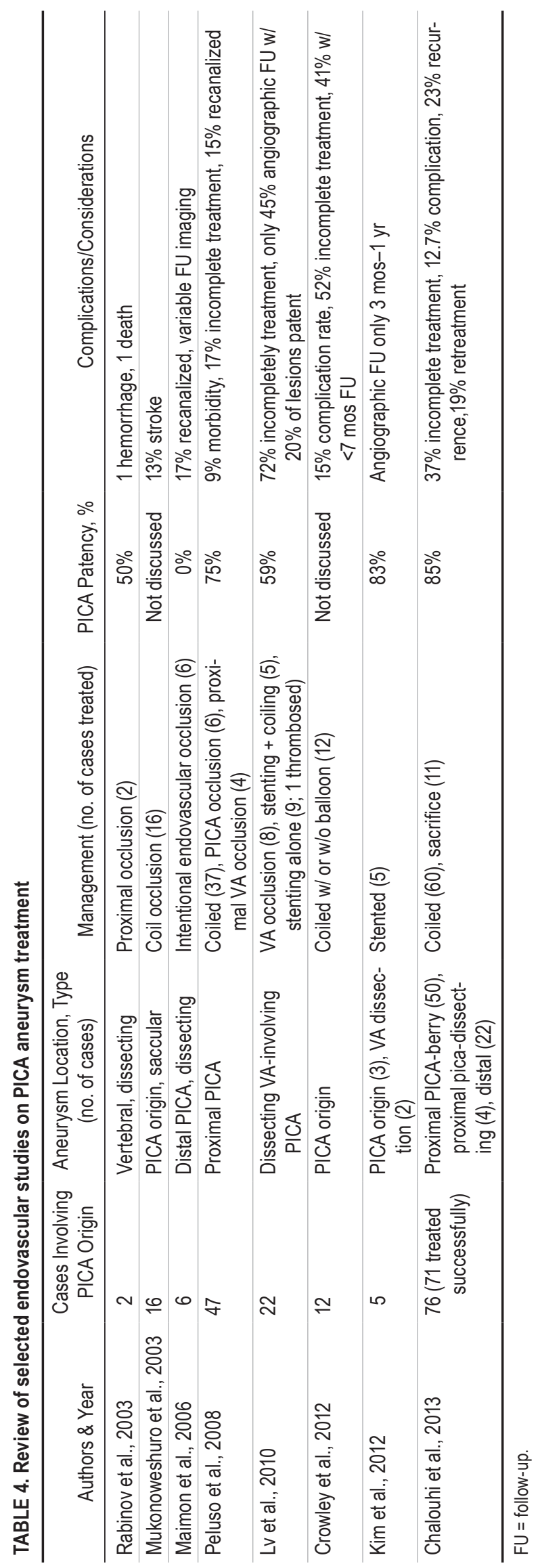

J Neurosurg Volume 124 • May 2016 


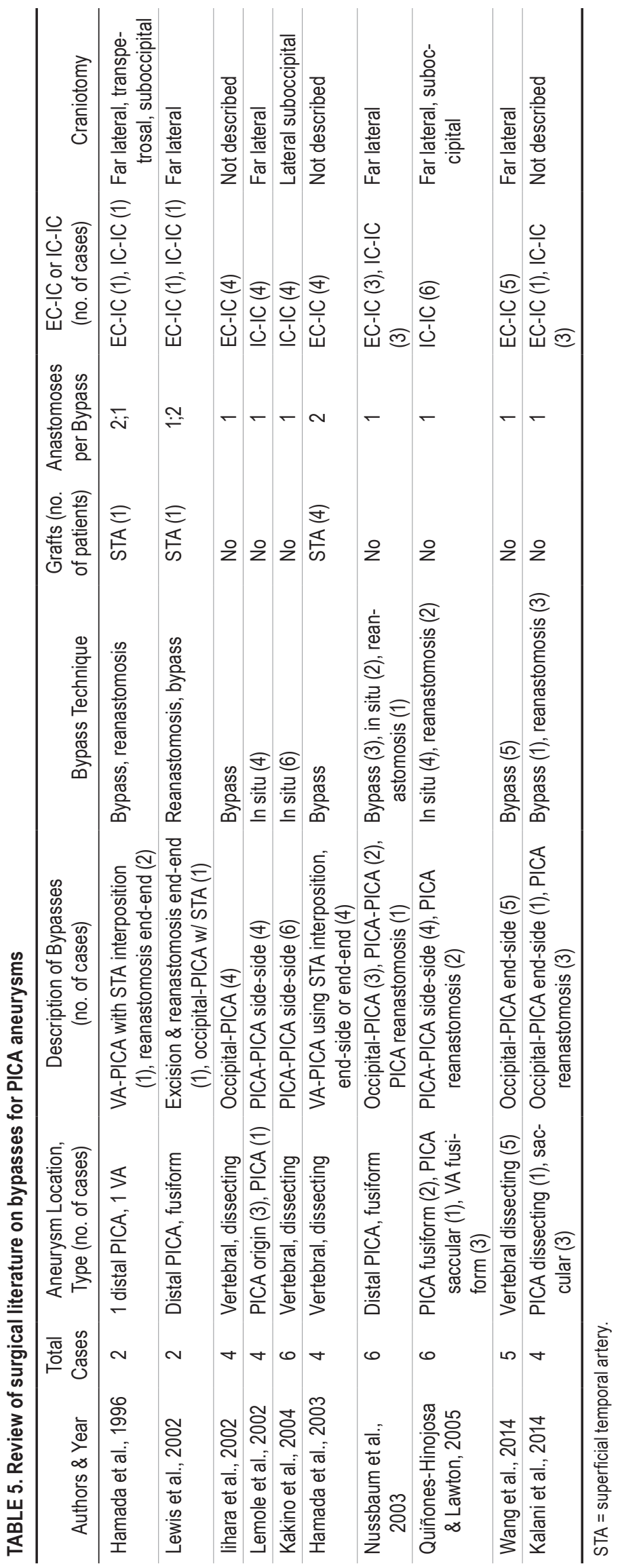


ample, a PICA-PICA bypass may not be the optimal choice when the donor PICA is hypoplastic. Similarly, PICA reanastomosis in the $\mathrm{p} 4$ segment may not seem necessary due to its distal location, but in both of our cases this parent artery was hyperplastic and the p4 segment had both ipsi- and contralateral territories to supply. Therefore, the angiographic anatomy may provide bypass indications and planning information preoperatively, but many of these decisions were finalized intraoperatively after inspecting the PICA caliber and the presence of direct medullary perforators.

\section{The Challenge of IC-IC Bypass}

Our experience demonstrates the advantages of IC-IC bypass for complex PICA aneurysms, but the adoption of such a practice appears limited (Table 5). Published surgical series with PICA bypass are small, with 43 total patients and an even split between the occipital artery-to-PICA and IC-IC bypasses (Table 5). ${ }^{5-9,13,14,19,22,29}$ PICA-PICA bypass was the most common IC-IC bypass, and only 4 cases of PICA reimplantation were reported., ${ }^{4,12,20,26}$ The limited application of IC-IC bypass may be explained by the rarity of dysmorphic PICA aneurysms, but there are other reasons that deserve consideration. First, these are technically challenging bypasses. The occipital artery-to-PICA bypass is a variation of the familiar superficial temporal artery-tomiddle cerebral artery bypass with an extracranial donor artery, end-to-side anastomosis, and anastomosis site that can be chosen for its accessibility. In contrast, IC-IC bypasses require more difficult and less familiar techniques, like the intraluminal sewing needed for the deep suturing of side-to-side PICA-PICA anastomosis, gentle handling of the endothelial surfaces, and careful accounting of 4 arterial walls. The anastomosis site is dictated by the anatomy and may not be at the most convenient location. For example, there may be only 1 reimplantation site on the proximal VA, which may be covered by the accessory nerve or coursing downward at a steep angle, making the anastomosis awkward and uncomfortable. Although the mechanics of anastomosis are the same with EC-IC and IC-IC bypass, the flexibilities that come with EC-IC bypass make it easier and are often lost with IC-IC bypass.

Second, the use of an intracranial donor artery raises concerns about its safety. The contralateral PICA in PICAPICA bypass is endangered during cross-clamping and there is a chance that the bypass could occlude. Similarly, PICA reimplantation and VA grafting require VA arteriotomy and anastomosis, which, if it occludes, might compromise basilar circulation. In addition, medullary perforators originating from the proximal PICA might constrain the artery's transposition inferiorly. Reanastomosis of the PICA may elicit similar concerns about perforators if they originate from the excised aneurysmal segment. In contrast, an occipital artery stirs no concerns and makes the EC-IC bypass option more comfortable.

Third, and most important, IC-IC bypass has an element of uncertainty. Transecting the efferent PICA branch from an aneurysm in order to reimplant it, or excising the aneurysmal segment to reanastomose the PICA, both require a judgment or leap of faith that the deconstructive maneuver can be reconstructed without knowing it in advance. What if the PICA cannot be moved all the way to the donor site on the VA because of a tethering medullary branch? What if the ends of the artery cannot be reapproximated after excising all of the aneurysm? Questions that can usually be answered in advance with EC-IC bypass are unanswerable in advance with IC-IC bypass. Therefore, adopting an IC-IC bypass approach requires more than just executing technical maneuvers, but also a willingness to perform a difficult procedure with certain risk and uncertainty.

\section{Conclusions}

PICA aneurysms are ideally suited to IC-IC bypass and obviate the need for EC-IC bypass. The PICA-PICA bypass can be used for PICA aneurysms on any segment except the p4 and is perhaps the most versatile. Reanastomosis can be used with PICA aneurysms beyond the P1 segment. Due to its depth, proximity to lower cranial nerves, increased morbidity, and decreased patency, reimplantation is reserved as an alternative when these bypass options are unfavorable. Interposition grafts are rarely needed for PICA aneurysms. A constructive approach that preserves the PICA with direct clipping or replaces flow with a bypass when sacrificed should remain an alternative to deconstructive PICA occlusion and endovascular coiling when complete aneurysm occlusion is unlikely.

\section{References}

1. Bertalanffy H, Sure U, Petermeyer M, Becker R, Gilsbach JM: Management of aneurysms of the vertebral artery-posterior inferior cerebellar artery complex. Neurol Med Chir (Tokyo) 38 Suppl:93-103, 1998

2. Chalouhi N, Jabbour P, Starke RM, Tjoumakaris SI, Gonzalez LF, Witte S, et al: Endovascular treatment of proximal and distal posterior inferior cerebellar artery aneurysms. J Neurosurg 118:991-999, 2013

3. Crowley RW, Albuquerque FC, Ducruet AF, Williamson RW, McDougall CG: Technical considerations in the endovascular management of aneurysms of the posterior inferior cerebellar artery. Neurosurgery 71:ons204-ons218, 2012

4. Durward QJ: Treatment of vertebral artery dissecting aneurysm by aneurysm trapping and posterior inferior cerebellar artery reimplantation. Case report. J Neurosurg 82:137-139, 1995

5. Hamada J, Kai Y, Morioka M, Yano S, Todaka T, Ushio Y: Multimodal treatment of ruptured dissecting aneurysms of the vertebral artery during the acute stage. J Neurosurg 99:960-966, 2003

6. Hamada J, Nagahiro S, Mimata C, Kaku T, Ushio Y: Reconstruction of the posterior inferior cerebellar artery in the treatment of giant aneurysms. Report of two cases. J Neurosurg 85:496-499, 1996

7. Iihara K, Sakai N, Murao K, Sakai H, Higashi T, Kogure S, et al: Dissecting aneurysms of the vertebral artery: a management strategy. J Neurosurg 97:259-267, 2002

8. Kakino S, Ogasawara K, Kubo Y, Otawara Y, Tomizuka N, Suzuki M, et al: Treatment of vertebral artery aneurysms with posterior inferior cerebellar artery-posterior inferior cerebellar artery anastomosis combined with parent artery occlusion. Surg Neurol 61:185-189, 2004

9. Kalani MY, Ramey W, Albuquerque FC, McDougall CG, Nakaji P, Zabramski JM, et al: Revascularization and aneurysm surgery: techniques, indications, and outcomes in the endovascular era. Neurosurgery 74:482-498, 2014

10. Kim MJ, Chung J, Kim SL, Roh HG, Kwon BJ, Kim BS, et al: Stenting from the vertebral artery to the posterior inferior 
cerebellar artery. AJNR Am J Neuroradiol 33:348-352, 2012

11. Lawton MT, Quiñones-Hinojosa A, Chang EF, Yu T: Thrombotic intracranial aneurysms: classification scheme and management strategies in 68 patients. Neurosurgery 56:441-454, 2005

12. Lee SY, Sekhar LN: Treatment of aneurysms by excision or trapping with arterial reimplantation or interpositional grafting. Report of three cases. J Neurosurg 85:178-185, 1996

13. Lemole GM Jr, Henn J, Javedan S, Deshmukh V, Spetzler RF: Cerebral revascularization performed using posterior inferior cerebellar artery-posterior inferior cerebellar artery bypass. Report of four cases and literature review. J Neurosurg 97:219-223, 2002

14. Lewis SB, Chang DJ, Peace DA, Lafrentz PJ, Day AL: Distal posterior inferior cerebellar artery aneurysms: clinical features and management. J Neurosurg 97:756-766, 2002

15. Liew D, Ng PY, Ng I: Surgical management of ruptured and unruptured symptomatic posterior inferior cerebellar artery aneurysms. Br J Neurosurg 18:608-612, 2004

16. Lv X, Jiang C, Li Y, Wu Z: Clinical outcomes of ruptured and unruptured vertebral artery-posterior inferior cerebellar artery complex dissecting aneurysms after endovascular embolization. AJNR Am J Neuroradiol 31:1232-1235, 2010

17. Maimon S, Saraf-Lavi E, Rappaport ZH, Bachar G: Endovascular treatment of isolated dissecting aneurysm of the posterior inferior cerebellar artery. AJNR Am J Neuroradiol 27:527-532, 2006

18. Mukonoweshuro W, Laitt RD, Hughes DG: Endovascular treatment of PICA aneurysms. Neuroradiology 45:188-192, 2003

19. Nussbaum ES, Mendez A, Camarata P, Sebring L: Surgical management of fusiform aneurysms of the peripheral posteroinferior cerebellar artery. Neurosurgery 53:831-835, 2003

20. O'Shaughnessy BA, Getch CC, Bowman RM, Batjer HH: Ruptured traumatic vertebral artery pseudoaneurysm in a child treated with trapping and posterior inferior cerebellar artery reimplantation. Case report and review of the literature. J Neurosurg 102 (2 Suppl):231-237, 2005

21. Peluso JP, van Rooij WJ, Sluzewski M, Beute GN, Majoie $\mathrm{CB}$ : Posterior inferior cerebellar artery aneurysms: incidence, clinical presentation, and outcome of endovascular treatment. AJNR Am J Neuroradiol 29:86-90, 2008

22. Quiñones-Hinojosa A, Lawton MT: In situ bypass in the management of complex intracranial aneurysms: technique application in 13 patients. Neurosurgery 57 (1 Suppl):140-145, 2005
23. Rabinov JD, Hellinger FR, Morris PP, Ogilvy CS, Putman $\mathrm{CM}$ : Endovascular management of vertebrobasilar dissecting aneurysms. AJNR Am J Neuroradiol 24:1421-1428, 2003

24. Rodríguez-Hernández A, Zador Z, Rodríguez-Mena R, Lawton MT: Distal aneurysms of intracranial arteries: application of numerical nomenclature, predilection for cerebellar arteries, and results of surgical management. World Neurosurg 80:103-112, 2013

25. Sanai N, Zador Z, Lawton MT: Bypass surgery for complex brain aneurysms: an assessment of intracranial-intracranial bypass. Neurosurgery 65:670-683, 2009

26. Shin YS, Kim SY, Cho KH, Cho KG: Treatment of vertebral artery dissecting aneurysms presenting with progressive myelopathy. J Clin Neurosci 11:896-898, 2004

27. Sughrue ME, Saloner D, Rayz VL, Lawton MT: Giant intracranial aneurysms: evolution of management in a contemporary surgical series. Neurosurgery 69:1261-1271, 2011

28. Waldron JS, Halbach VV, Lawton MT: Microsurgical management of incompletely coiled and recurrent aneurysms: trends, techniques, and observations on coil extrusion. Neurosurgery 64 (5 Suppl 2):301-317, 2009

29. Wang C, Shi XE, Wang J, Wang B, Tang Z: Long-term outcomes of trapping vertebral artery-posterior inferior cerebellar artery dissecting aneurysms after revascularization. Neurol India 62:15-18, 2014

\section{Disclosures}

Dr. Lawton has a financial relationship with Mizuho America, Inc.

\section{Author Contributions}

Conception and design: Lawton, Abla. Acquisition of data: all authors. Analysis and interpretation of data: all authors. Drafting the article: Lawton, Abla. Critically revising the article: Lawton. Reviewed submitted version of manuscript: Lawton, Abla. Approved the final version of the manuscript on behalf of all authors: Lawton. Statistical analysis: Lawton, Abla, McDougall. Administrative/technical/material support: Lawton. Study supervision: Lawton.

\section{Correspondence}

Michael T. Lawton, Department of Neurosurgery, University of California, San Francisco, 505 Parnassus Ave., M780, San Francisco CA 94143. email: Michael.Lawton@ucsf.edu. 